\title{
Background Oriented Schlieren Implementation in a Jet-Surface Interaction Test
}

\author{
Michelle M. Clem * , Clifford A. Brown ${ }^{\dagger}$ and Amy F. Fagan ${ }^{\ddagger}$ \\ NASA Glenn Research Center, Cleveland, OH, 44135, USA
}

\begin{abstract}
Many current and future aircraft designs rely on the wing or other aircraft surfaces to shield the engine noise from observers on the ground. However the available data regarding how a planar surface interacts with a jet to shield and/or enhance the jet noise are currently limited. Therefore, the Jet-Surface Interaction Tests supported by NASA's Fundamental Aeronautics Program's Fixed Wing Project were undertaken to supply experimental data covering a wide range of surface geometries and positions interacting with high-speed jet flows in order to support the development of noise prediction methods. Phase 1 of the Test was conducted in the Aero-Acoustic Propulsion Laboratory at NASA Glenn Research Center and consisted of validating noise prediction schemes for a round nozzle interacting with a planar surface. Phased array data and far-field acoustic data were collected for both the shielded and reflected sides of the surface. Phase 1 results showed that the broadband shock noise was greatly reduced by the surface when the jet was operated at the overexpanded condition, however, it was unclear whether this reduction was due a change in the shock cell structure by the surface. In the present study, Background Oriented Schlieren is implemented in Phase 2 of the Jet-Surface Interaction Tests to investigate whether the planar surface interacts with the high-speed jet flow to change the shock cell structure. Background Oriented Schlieren data are acquired for under-expanded, ideally-expanded, and over-expanded flow regimes for multiple axial and radial positions of the surface at three different plate lengths. These data are analyzed with far-field noise measurements to relate the shock cell structure to the broadband shock noise produced by a jet near a surface.
\end{abstract}

\section{Nomenclature}

$\Delta x \quad$ appartent image displacement/distance traveled by the refracted light angle through which light is refracted

observation angle relative to the upstream jet axis

speed of sound at jet exit condtions

$\begin{array}{ll}c_{j} & \text { speed of sound at } \\ D_{j} & \text { jet exit diameter }\end{array}$

$h \quad$ radial distance from jet centerline to surface

$M_{D} \quad$ design Mach number

$M_{i d} \quad$ ideally expanded Mach number

$M_{j} \quad$ gas dynamic jet Mach number $\left(M_{j}=U_{j} / c_{j}\right)$

$r \quad$ radial distance to the jet centerline

$S \quad$ schlieren object

$S t_{D_{j}} \quad$ Strouhal Frequency (normalized by the jet exit diameter)

$x$ axial location relative to the jet exit plane

$x_{T E} \quad$ axial distance from jet exit to surface trailing edge

AAPL Aero-Acoustic Propulsion Laboratory

BBSN Broadband Shock Noise

\footnotetext{
${ }^{*}$ Research Engineer, Optical Instrumentation and NDE Branch, 21000 Brookpark Rd.

${ }^{\dagger}$ Research Engineer, Acoustics Branch, 21000 Brookpark Rd., AIAA Member.

${ }^{\ddagger}$ Research Engineer, Optical Instrumentation and NDE Branch, 21000 Brookpark Rd., AIAA Senior Member.
} 


$\begin{array}{ll}\text { BOS } & \text { Background Oriented Schlieren } \\ \text { JSIT } & \text { Jet-Surface Interaction Tests } \\ \text { NPR } & \text { Nozzle Pressure Ratio } \\ \text { PIV } & \text { Particle Image Velocimetry } \\ \text { PSD } & \text { Power Spectral Density } \\ \text { SHJAR } & \text { Small Hot Jet Acoustic Rig }\end{array}$

\section{Introduction}

The noise created by a supersonic aircraft is a primary concern in the design of future military and high-speed commercial aircraft. At cruise, sonic boom is the largest noise source related to supersonic flight. However, engine exhaust (jet) noise remains the dominant noise source at take-off and landing. Furthermore, tighter integration of the engine and airframe to reduce sonic boom puts the engine exhaust in close proximity to the aircraft surfaces leading to jet-surface interaction noise. However, these surfaces, correctly placed, may be used to shield observers on the ground from some of the jet noise. This seems particularly appropriate for mitigation of broadband shock noise (BBSN) which is dominant at upstream angles where the surface shielding effect is the greatest.

Broadband shock noise is created when turbulent flow structures interact with the shock cells in a supersonic jet plume. The amplitude and frequency characteristics of BBSN are highly dependent on the strength, number, and location of the shock cells. In a single stream jet, the shock cell structure is dictated by the nozzle profile and nozzle pressure ratio (NPR). The shock cells, and therefore BBSN, can be minimized by operating a convergent-divergent nozzle at its design Mach number $\left(M_{D}\right)$. In practice, however, this requires a complex actuation and control system to continuously vary the nozzle area throughout the operating range. This situation becomes more complicated when the airframe surfaces are very near the engine exhaust. In these configurations, the high-speed flow may interact with the surface and ambient entrainment air to alter the shock cell structure, presenting a significant challenge to aircraft designers trying to meet noise regulations.

The current generation of available engine exhaust noise prediction tools and methods have been developed to predict the noise of an isolated jet and are not generally applicable to configurations where the engine and airframe are tightly coupled. Moreover, quality experimental noise data with flow diagnostics suitable for developing and validating noise prediction methods for these configurations are limited. The Jet-Surface Interaction Tests (JSIT) are being supported by the NASA Fundamental Aeronautics Program, Fixed Wing Project to create an experimental database over a wide range of surface geometries and engine exhaust conditions to improve and validate the current generation of aircraft noise prediction tools. While these tests primarily focus on subsonic airport operations, a smaller set of data at supersonic flow conditions has been acquired to show the limits of the current prediction methods and to identify the challenges in extending them to supersonic flow regimes. These data include over-expanded, ideally expanded, and under-expanded jet exit conditions near a flat surface of varying length and distance from the nozzle centerline. Far-field noise data were acquired during Phase 1 of the JSIT in part to establish "regions of interest" for future testing. These data at supersonic engine exhaust conditions showed significant changes to the BBSN when a flat surface was near the exhaust plume. In particular, the surface effectively shielded the BBSN from an over-expanded jet when placed between the jet and observer. ${ }^{1,2}$ This is potentially a useful finding as most supersonic jets operate in the over-expanded regime when at low altitudes. Therefore, in Phase 2, Background Oriented Schlieren (BOS) was deployed to provide a better understanding of how the surface impacts the shock cell structure and, thus, affects the BBSN. BOS is an optical technique used to non-intrusively visualize density gradients, similar to classical schlieren and shadowgraph methods but with a much simpler and less costly setup. ${ }^{3}$ These images are coupled with the far-field noise data to give the developers of engine exhaust noise prediction methods some insight into how a solid surface affects BBSN.

\section{Test Setup and Data Systems}

\section{A. Test Setup}

The JSIT were conducted using the Small Hot Jet Acoustic Rig (SHJAR) located in the Aero-Acoustic Propulsion Laboratory (AAPL) at the NASA Glenn Research Center (GRC). The SHJAR is capable of 
supplying air at flow rates up to $6 \mathrm{lbm} / \mathrm{s}$ to a single-steam nozzle. A hydrogen burning combustor is used to simulate core exhaust at temperatures up to $1300^{\circ} \mathrm{F}$. Flow conditioning and a line-of-sight muffler are used to achieve a clean and quiet flow at jet exit Mach numbers down to $M_{a}=0.35$. The AAPL is covered with wedges to provide an anechoic environment at frequencies above $200 \mathrm{~Hz}$. Additional information on the SHJAR, including performance validation data, can be found in Ref. 5 and Ref. 6 .

Figure 1 shows the configuration used for these tests was formed using a flat plate near a round nozzle. The plate was allowed to move on an automated traverse between $0.5 \leq h / D_{j} \leq 5$ in the radial direction. The plate was assembled using multiple pieces of $0.5 \mathrm{in}$ thick aluminum to allow 6 lengths between $2 \leq$ $x_{T E} / D_{j} \leq 15$. Note that while every surface position was tested for far-field noise, only a subset were tested with BOS. In order to minimize the thickness, the surface trailing edge, shown in Fig. 2, was cut from a $0.25 \mathrm{in}$ thick aluminum plate with a beveled downstream edge and attached to the $0.5 \mathrm{in}$ piece.

"Shielded" Observer

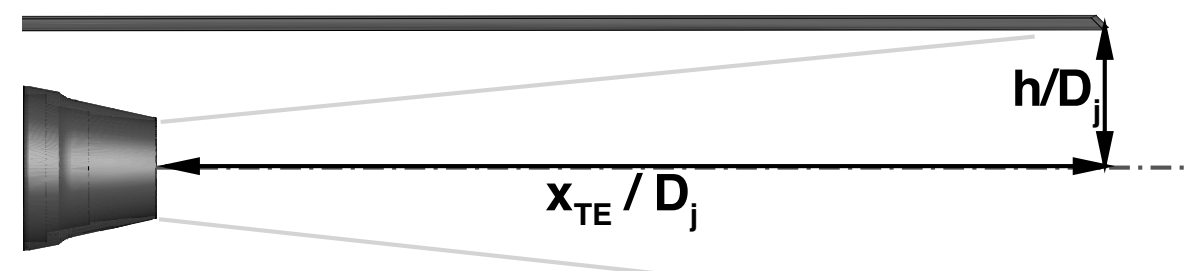

"Reflected" Observer

Figure 1. Schematic showing the configuration tested with the nomenclature used to describe the surface and observed locations.

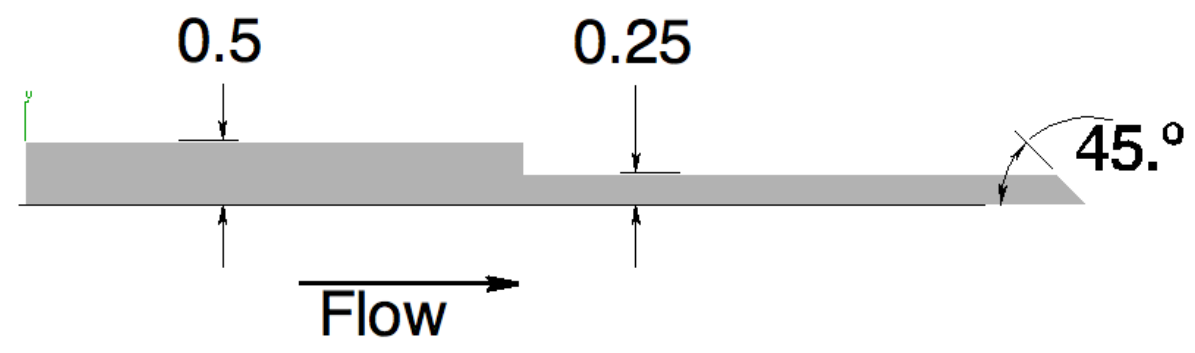

Figure 2. Schematic showing the surface trailing edge geometry.

A convergent-divergent nozzle was used for all the supersonic jet conditions, which is shown in Fig. 3. The design Mach number for this nozzle was $M_{d}=1.5$ although test data indicated that the minimum shock point occurred at a slightly lower value. Therefore the ideally expanded Mach number, $M_{i d}=1.48$, was used for $M_{d}$ for this nozzle. This nozzle was run at the over-expanded, ideally expanded, and underexpanded jet exit conditions shown in Table 1. To ensure the correct operating conditions, each point in Table 1, as defined by $M_{i d}$, was entered into a facility data acquisition system, which monitored all relevant rig temperatures and pressures once per second to compute the difference between the current and specified jet exit conditions (based on an L2-norm). The jet condition was required to be within $0.5 \%$ of the specified value throughout the acquisition for an acoustic point to be accepted. The same system was used during the BOS portion of the test but the accuracy requirement was relaxed slightly due to the longer acquisition times. The facility data acquisition system also acquired and stored the ambient conditions for later reference and data corrections. 


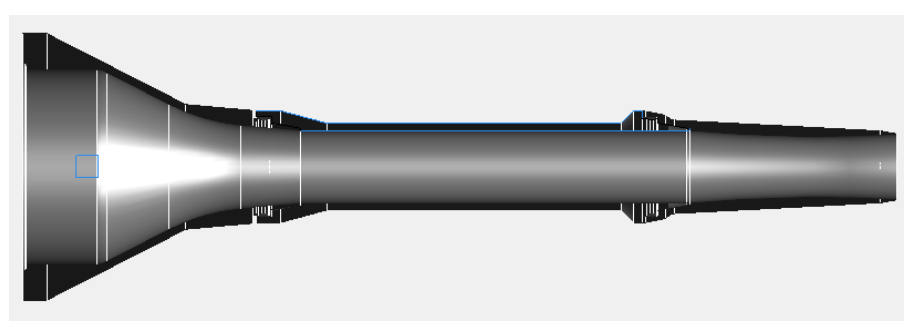

Figure 3. Cut view of the convergent-divergent nozzle used for the JSIT supersonic jet conditions.

Table 1. Supersonic jet exit conditions for the data acquired using the convergent-divergent nozzle with $M_{d}=1.48\left(D_{j}=2.0 \mathrm{in}\right)$.

\begin{tabular}{|c|c|c|c|c|c|}
\hline Regime & Setpoint & $\begin{array}{c}\mathrm{NPR} \\
P_{j} / P_{a}\end{array}$ & $\begin{array}{c}T_{s, j} / T_{a} \\
V_{i d} / c_{a}\end{array}$ & $\begin{array}{c}M_{i d} \\
\mathrm{~kg} / \mathrm{s}\end{array}$ & Mass Flow \\
\hline Over-expanded & 11606 & 2.750 & 0.750 & 1.29 & 1.12 \\
\hline Ideally expanded & 11610 & 3.595 & 0.694 & 1.48 & 1.50 \\
\hline Under-expanded & 11617 & 4.312 & 0.660 & 1.61 & 1.79 \\
\hline
\end{tabular}

\section{B. Acoustic Data Acquisition and Processing}

Far-field noise data were acquired for each surface configuration and jet exit condition. An array of 24 microphones (Bruel \& Kjaer type 4939) were placed on a $150 \mathrm{in}$ radius arc, centered on the nozzle exit, at $5^{\circ}$ intervals between $50^{\circ}$ (upstream) and $165^{\circ}$ (downstream). Bruel \& Kjaer Nexus units were used for signal conditioning and amplification. A DataMAX Instrumentation Recorder from R. C. Electronics was used to digitize the data at a $200 \mathrm{kHz}$ sample rate with a $90 \mathrm{kHz}$ low pass Nyquist filter. The time series data were then transformed by a standard Fast Fourier Transform routine using a $2^{14}$ point Kaiser window to give a frequency resolution of $12.21 \mathrm{~Hz}$. The data were then corrected for the individual microphone freefield response on a frequency by frequency basis using the current calibration supplied by the manufacturer. Finally, a correction was applied to account for atmospheric attenuation and scaled to a distance of $100 D_{j}$ from nozzle exit assuming spherical spreading of sound.

Acoustic data were acquired with the surface in both the shielded and reflected positions (Fig. 1). However, the jet-surface setup described in section A was modified for acoustic testing in the shielded position to ensure the surface was semi-infinite in the upstream and vertical directions so that sound could not pass. The surface was backed by a curtain made from medium-duty welding blankets that was attached to the face of the jet rig at the upstream end and to a support behind the surface at the downstream end. The effectiveness of this arrangement was verified by far-field and phased-array measurements.

\section{Background Oriented Schlieren Data Acquisition and Processing}

Background Oriented Schlieren is based on background distortion or an apparent movement of the background when imaged through a density field onto a detector plane. Figure 4 illustrates the principle of BOS. An incoherent light source uniformly illuminates a background composed of a high-contrast random pattern that is imaged by a camera and lens system. If applicable, the background can be back-illuminated as shown in Fig. 4. Without any refractive index gradient (denoted ' $S$ ' ) in the optical path, light will travel from the background to the camera undisturbed. The dashed green line, along the optical axis illustrates an individual light ray traveling undisturbed from a point on the background to the camera in the case without a refractive index gradient present. When acquiring an image under this condition, the background will look undistorted. A refractive index gradient in the path causes light to refract. The solid green line in Fig. 4 shows the light ray being refracted by the gradient at ' $S$ '. Now when the background is imaged through the non-uniform density field, points on the background will appear shifted by ' $\Delta x$ ' at the detector plane. Therefore, when acquiring BOS data it is necessary to take two images: a reference image prior to the refractive index gradient being present and an image after the light has refracted through the density field. For maximum sensitivity the refractive index gradient should occur halfway between the camera and 
the background. ${ }^{7}$

The shift between the two acquired images caused by the refracted light can be calculated by correlation methods. Existing algorithms that have already been developed for particle image velocimetry (PIV) are used to determine the background image displacements within small sub-regions across the image. ${ }^{8}$ Unique to BOS, the density gradient in two orthogonal directions in the plane of the background can be distinguished from one another.

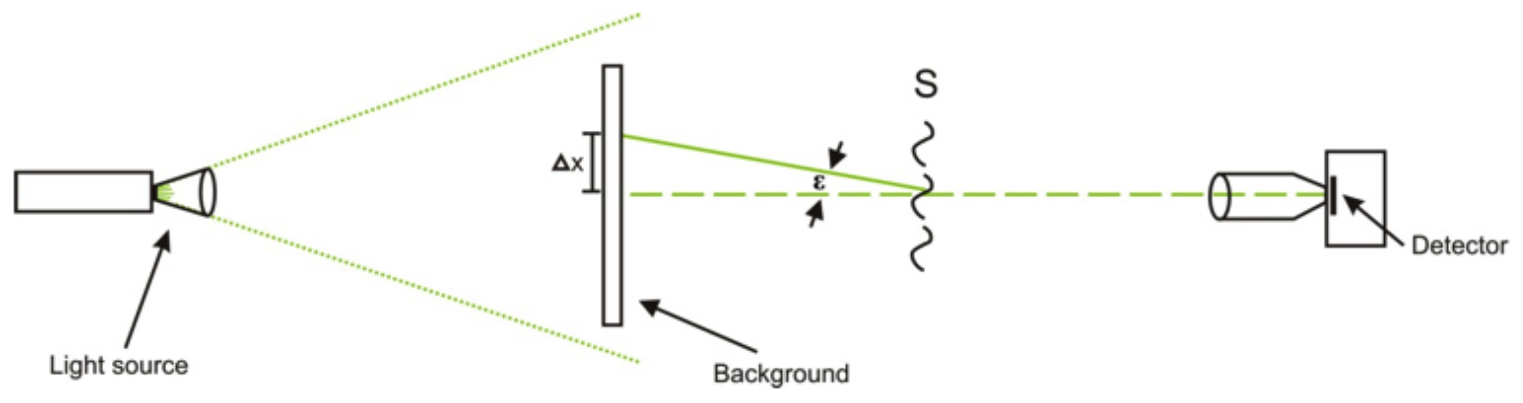

Figure 4. Illustration of the principle of BOS, showing light passing through a schlieren object $(S)$ resulting in a displacement of $\Delta x$ which appears as a pixel shift in the acquired images.

The BOS system was configured specifically for the demands of the jet-surface geometry. A schematic of the BOS setup, looking into the plane of the nozzle, is shown in Fig. 5. A 10 W, $530 \mathrm{~nm}$ LED is used as the incident light source for the system. The LED is mounted $0.6 \mathrm{~m}$ behind the background in order to uniformly back-illuminate the $0.9 \mathrm{~m} \times 0.7 \mathrm{~m}$ target, consisting of a MATLAB generated high contrast random dot pattern. The dot pattern is composed of $0.7 \mathrm{~mm}$ diameter transparent dots on a black background and has approximately a $32 \%$ fill ratio. The size of the dots in the pattern was chosen such that one dot would span 2-3 pixels after being imaged through the optical system. The pattern was printed onto clear film and mounted onto Plexiglas, which enabled the back illumination. The incident light traveling through the flow was focused by a Nikon $105 \mathrm{~mm}$ lens onto the $36 \mathrm{~mm} \times 24 \mathrm{~mm}$ detector of a Princeton Instruments EC11000 scientific-grade CCD camera. The resulting 12-bit images were captured at an exposure time of $275 \mathrm{~ms}$ with a resolution of 4008 pixels $\times 2672$ pixels, consisting of $9 \mu \mathrm{m}^{2}$ pixels. The large detector size facilitated achievement of the desired $0.45 \mathrm{~m} \times 0.35 \mathrm{~m}$ field of view within the facility constraints. The background was placed at a distance of $2.8 \mathrm{~m}$ from the focusing lens of the camera. The experiment was designed so that the nozzle was located a distance of $1.4 \mathrm{~m}$ (halfway) between the background and the camera lens.

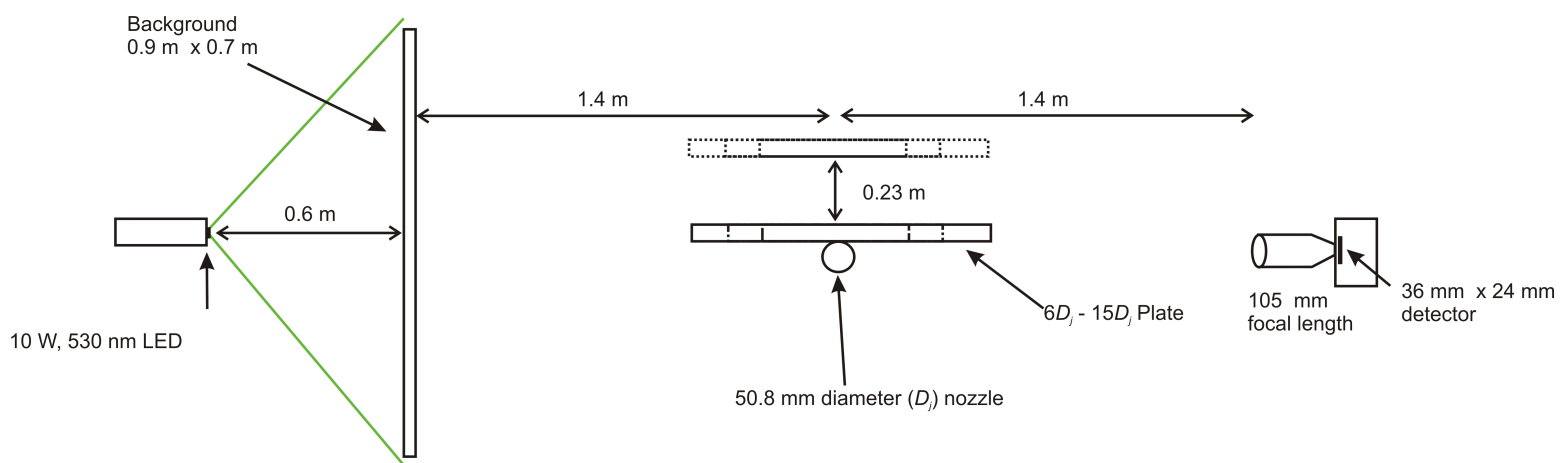

Figure 5. Schematic of the BOS setup, looking into the plane of the nozzle.

The BOS setup was mounted on an axial traversing system and was translated downstream with an approximate range of $0 \leq x / D_{j} \leq 20$. BOS reference and data images were acquired for each appropriate combination of axial and radial positions for each set point. Note that all of the reference data for each combination of axial and radial positions were acquired during no flow conditions prior to starting the flow each day, while the image data was acquired upon reaching the desired test conditions. Reference image data were not acquired in between new axial and radial combinations due to time constraints. Therefore, there is a large time lag between reference data and flow data during which physical aspects of the background 
may have changed, such as thermal growth, shrinkage of the background material, or slight changes in the the physical location of the background and/or the surface relative to the camera due to movement of the BOS system and surface on such large traversing mechanisms. These changes are the largest source of error in the displacement measurements. The image displacements, in both the axial $(x)$ and radial $(r)$ directions, between the corresponding BOS reference and data images were calculated using PIV software. The displacements were plotted as a contour diagram in the cartesian coordinate system, which enabled visualization of the density gradients in the axial and radial directions. In all of the data presented here, the magnitude of the resultant $x$ and $r$ image displacement vectors are plotted in order to visualize the magnitude of the flow structures. All plots are presented with the same maximum and minimum displacement range so that the relative shock strength comparisons can be made. Actual scales are not shown since the displacement values do not have direct meaning. The BOS images have an averaged data value for every $8 x 8$ pixel subregion, resulting in a spatial resolution of $0.97 \mathrm{~mm}$ per sub-region.

\section{Results and Analysis}

\section{A. Isolated Jet}

There are two principle noise sources in an isolated supersonic jet: jet mixing noise and broadband shock noise. Jet mixing noise originates at the inherently unstable free-shear layer between two fluids moving at different velocities. As the fluids mix, Kelvin-Helmholtz instability waves grow and decay governing the formation and destruction of unsteady flow structures, or eddies. These flow structures range in size from the large-scale eddies, on the order of the jet diameter, that are responsible for transporting energy in the jet plume to the Kolmogorov microscales, where the turbulent kinetic energy dissipates through viscous forces. Figure 6 shows noise spectra for the over-expanded, ideally expanded, and under-expanded flow regimes at $\theta=60^{\circ}$ and $\theta=90^{\circ}$. The wide range of turbulent scales is responsible for the characteristic broadband jet mixing noise illustrated by the ideally expanded spectra. Experimental and theoretical research has shown that the jet mixing noise scales with the velocity difference across the shear layer. ${ }^{10}$ When the turbulent eddies pass through the shock cells in an over-expanded or under-expanded jet, BBSN is generated, whose characteristic spectra are also shown in Fig. 6. When present, BBSN is a dominant noise source at upstream angles with a peak amplitude around $\theta=60^{\circ}$.

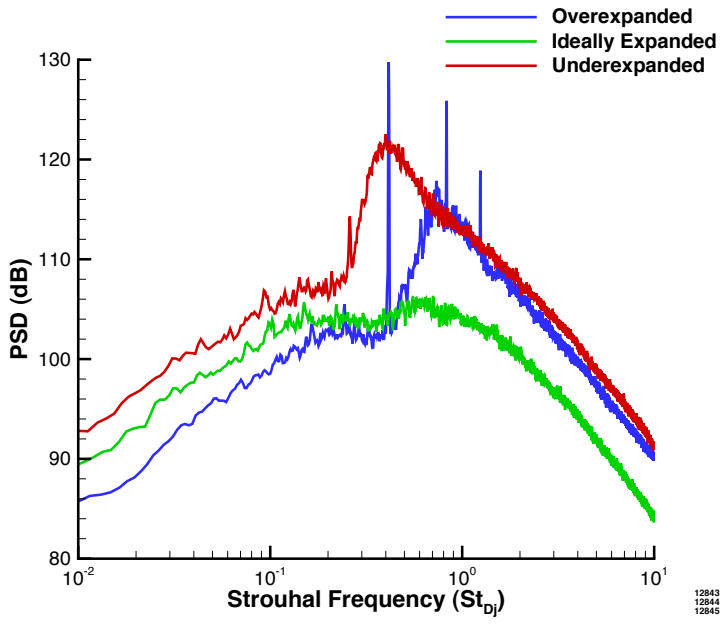

(a) $\theta=60^{\circ}$

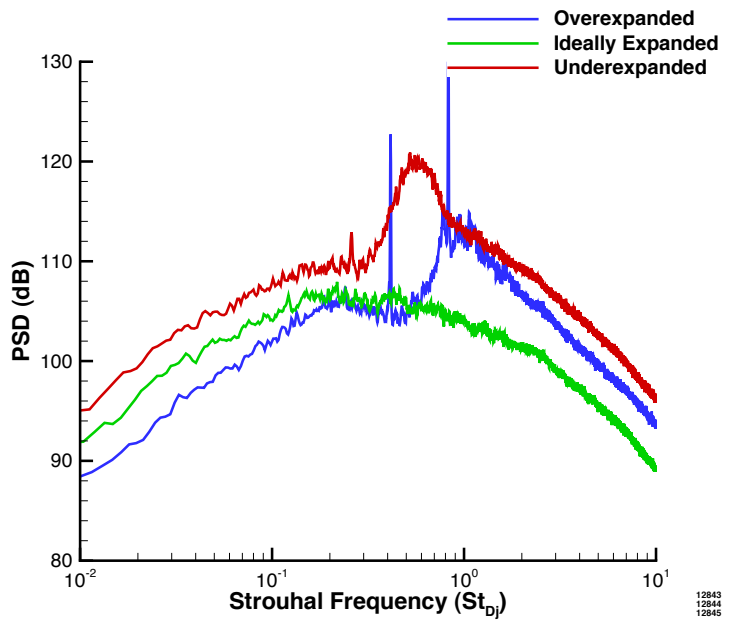

(b) $\theta=90^{\circ}$

Figure 6. Noise from the isolated jet operating over-expanded, ideally expanded, and under-expanded jet conditions.

The spectral characteristics of BBSN are a function of the shock cell strength, number, and spacing which is governed by the NPR. As a result, BBSN is a series of distributed noise sources. Figure 7 shows the shock cells, imaged using BOS, for an isolated jet operating in the three supersonic flow regimes. A perfectly expanded supersonic jet will not contain shock cells and, therefore, will not produce BBSN. In 
practice, however, it is extremely difficult to achieve a perfectly expanded jet (note faint diamond shocks in Fig. 7(b)). When over-expanded, the shock cells may start in the divergent section of the nozzle and remain fairly close to the nozzle exit (Fig. 7(a)). The under-expanded jet, however, has many more shock cells that extend to near $15 D_{j}$ (Fig. $7(\mathrm{c})$ ). By comparison, the shock cells in the under-expanded jet are also spaced farther apart than in the over-expanded case. These different shock cell structures are apparent in the far-field noise spectra. The ideally expanded jet has a small BBSN spectral hump due to the weak shock cells (Fig. 6(a)). Spectra at the over-expanded jet condition have a BBSN peak around $S t_{D j}=1$ with multiple tones that may arise from a shock cell resonance in the nozzle divergent section. ${ }^{13}$ The shock cell structure in the under-expanded jet produces BBSN that has a lower peak frequency $\left(S t_{D j}=0.4\right)$ and significantly higher amplitude than the over-expanded jet.

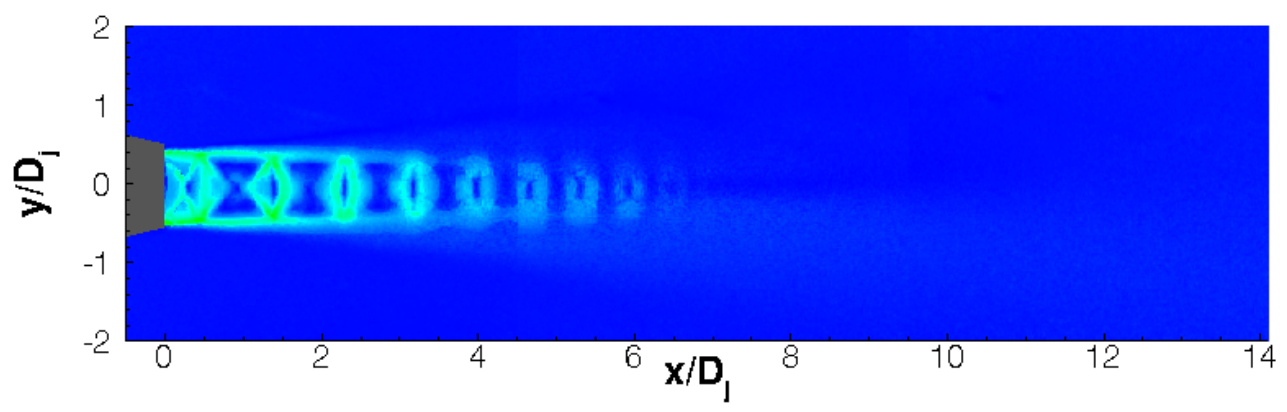

(a) Over-expanded

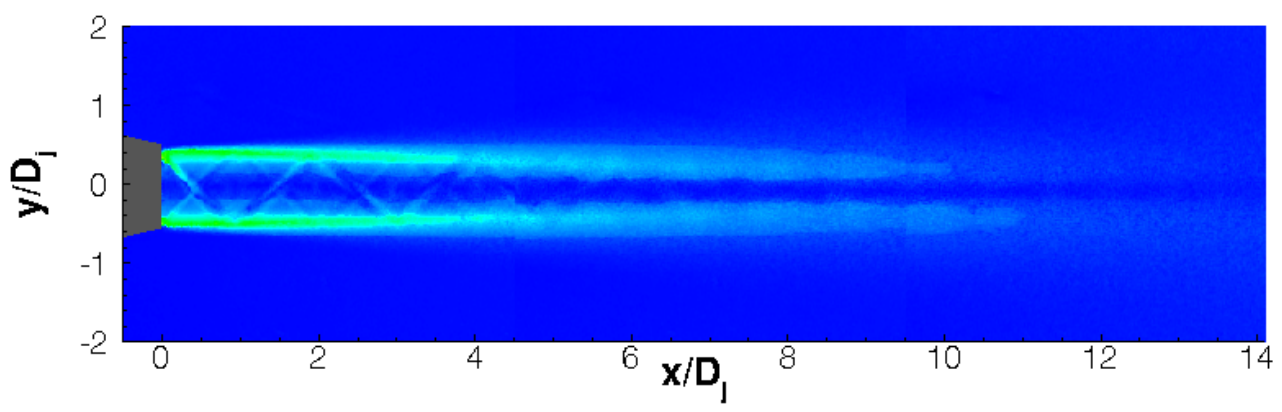

(b) Ideally Expanded

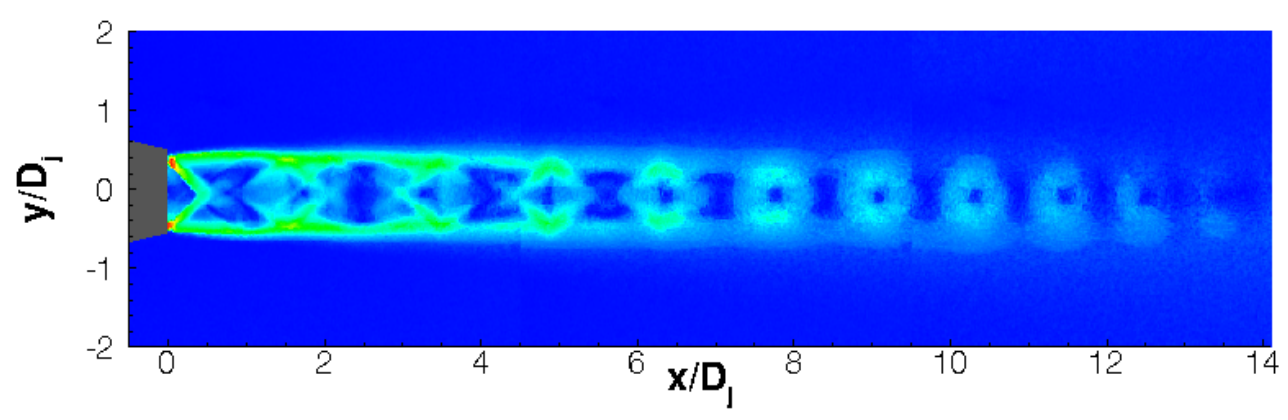

(c) Under-expanded

Figure 7. BOS showing the shock cell structure in an isolated over-expanded, ideally expanded, and under-expanded jet conditions.

\section{B. Jet Near a Surface}

\section{Effect of Surface Length}

Broadband shock noise and jet mixing noise are the primary noise sources in an isolated supersonic jet. However, when the flow is near a solid surface, jet-surface interaction noise sources may also become present. 
These noise sources, which include surface loading noise ('scrubbing') and trailing edge noise ('scattering'), are characterized as a low frequency augmentation in the noise spectrum. ${ }^{1,9,11}$ Jet-surface interaction noise is often the dominant source in a subsonic jet, however a surface near an isolated supersonic jet operating off design may affect the BBSN, making it the dominant noise source. Figure 8 shows noise measured from the jet operating in each flow regime near the peak radiation angle for BBSN $\left(\theta=60^{\circ}\right)$ with a surface at $x_{T E} / D_{j}=10$ and $h / D_{j}=0.75$. Noise spectra for the reflected and shielded observers are compared to the noise spectra from an isolated supersonic jet in each flow regime. At the ideally expanded condition shown in Fig. 8(b), the spectra is similar to that measured in a subsonic jet where the jet-surface interaction noise is significant at low frequencies and the jet mixing noise is dominant at high frequencies. The spectral peak around $S t_{D j}=0.05$ at the ideally expanded jet condition is due to jet-surface interaction which, in this configuration, is mostly trailing edge noise. ${ }^{2}$ It can be seen that there is noise reflection and shielding at high frequencies due to the nearby surface. The ideally expanded noise spectra contains minimal BBSN consistent with the weak shock cells observed in the isolated jet in Fig. 7(b). However, spectra measured at the under-expanded jet condition shown in Fig. 8(c) show that the BBSN, rather than jet-surface interaction or jet mixing noise, is the dominant noise source for both the shielded and reflected observers. The very little difference between the sides is an indication that most of the BBSN is generated downstream of the surface, minimizing the surface shielding effect. Even the reflected jet noise at high frequencies is overwhelmed and does not appear in the spectra. Conversely, when the jet is operated in the over-expanded condition, shown in Fig. 8(a), the reflected and shielded observers experience very different noise levels . On the reflected side, the BBSN is similar to the isolated jet with some noise reflection. However, the surface effectively shields the BBSN and much of the high frequency jet mixing noise.

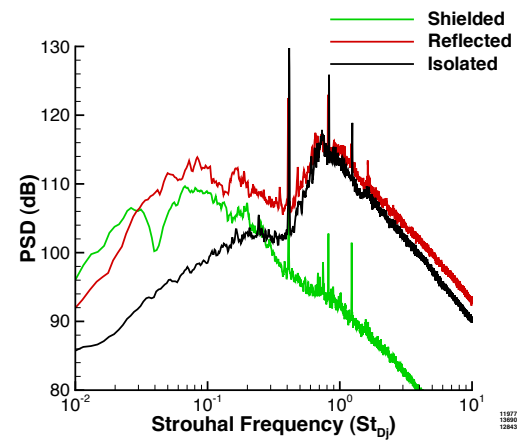

(a) Over-expanded

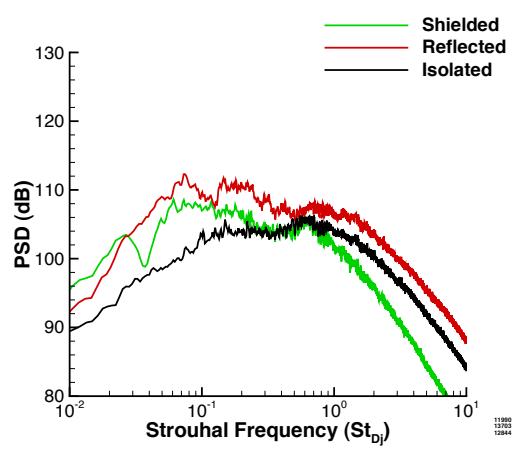

(b) Ideally Expanded

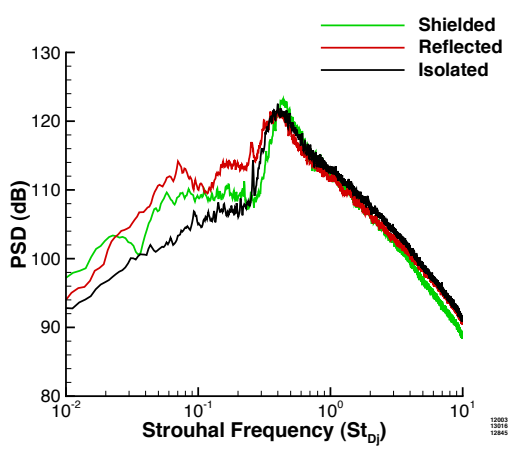

(c) Under-expanded

Figure 8. Noise spectra measured at over-expanded, ideally expanded, and under-expanded jet conditions near peak radiation angle for $\operatorname{BBSN}\left(\theta=60^{\circ}\right)$ with a surface a $x_{T E} / D_{j}=10$ and $h / D_{j}=0.75$.

It was observed in Fig. 7(a) that the shock cells move upstream in an over-expanded jet. It is believed that the surface shielding effect is increased the further upstream the shock cells remain. Conversely, it was observed in Fig.7(c) the shock cells move downstream in an under-expanded jet, which is believed to minimize the effect of the surface. For further illustration, the BOS images for the surface $x_{T E} / D_{j}=10$ and $h / D_{j}=0.75$, shown in Figs. 9(a) and 9(b), are compared to the corresponding far-field noise spectra that was shown in Fig. 8. Note, the nozzle and surface are depicted in the BOS image. The area directly above the plate consists of the plate mounting apparatus and can be ignored. The BOS image at the over-expanded jet condition (Fig. 9(a)) shows all of the shock cells are behind the surface for the shielded observer, consistent with the corresponding noise data showing a large shielding effect. However, the shock cells at the underexpanded jet condition (Fig. 9(b)) extend past the trailing edge of the same surface. Interestingly, many of the shock cells and particularly the strongest cells, are behind the surface to the shielded observer, yet the BBSN shielding was relatively small. In addition, it should be noted that although the $x_{T E} / D_{j}=10$ surface at $h / D_{j}=0.75$ appears to increase the strength of the upper portion of the shock cell structures directly underneath the plate, this cannot be concluded due to an artificial shift of the background that occurred during the time lag between when the no-flow reference images and on-condition images were acquired.

The shock cell structures imaged for the over-expanded isolated jet and the jet with the surface at $x_{T E} / D_{j}=10$ extend to approximately $x / D_{j}=7$ (Figs. 7(a) and 9(a), respectively). Figure 10(a) shows the shock cell structure and corresponding noise data for the over-expanded jet with a surface that only extends 


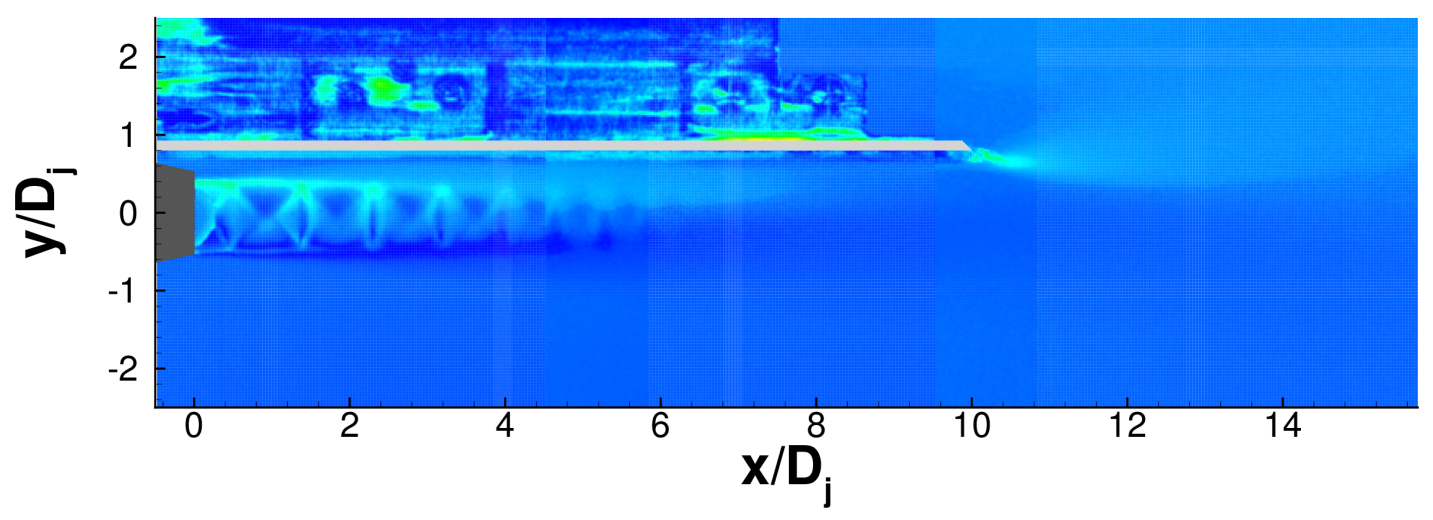

(a) Over-expanded

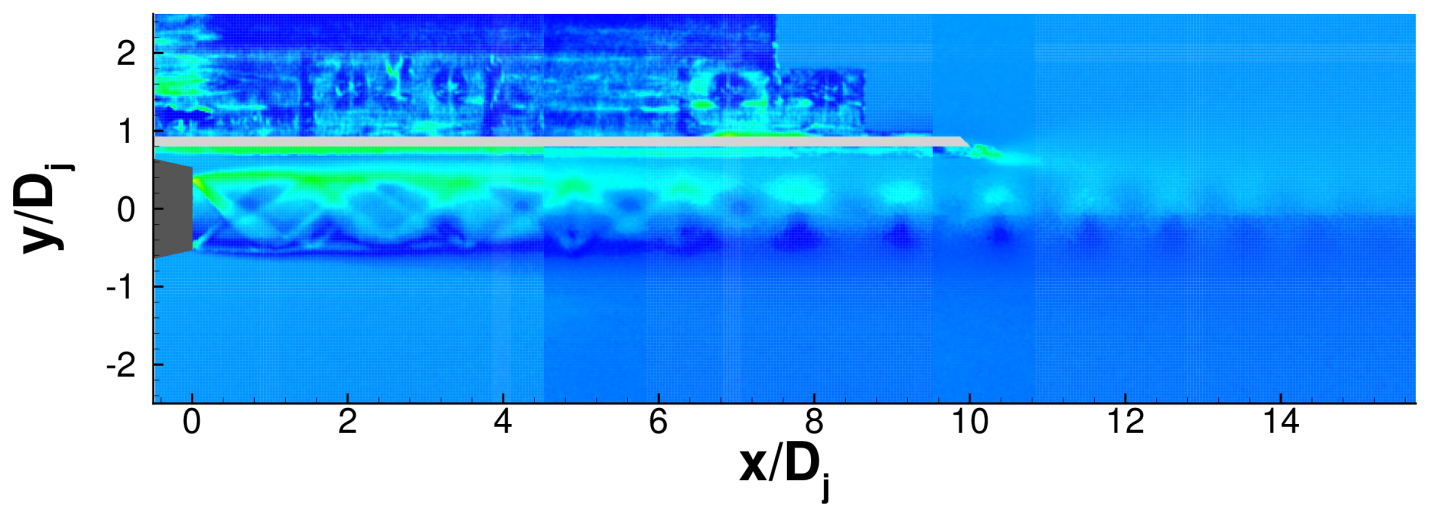

(b) Under-expanded

Figure 9. BOS showing the shock cell structure in an over-expanded and under-expanded jet with a surface at $x_{T E} / D_{j}=10$ and $h / D_{j}=0.75$.

to $x_{T E} / D_{j}=6$ at $h / D_{j}=0.75$. As in the previous BOS images at this over-expanded condition, the shock cells extend to around $x / D_{j}=7$. It is observed in the corresponding noise data, that the shorter surface is a less effective shield allowing some BBSN to reach the upstream end and, to a lesser extent, sideline observers. Note that the spectral hump associated with the BBSN is shifted to a higher frequency which is consistent with phased array measurements showing the high frequency BBSN sources farther downstream. ${ }^{2}$ Although, most of the shock cells are behind the $x_{T E} / D_{j}=6$ surface (to the shielded observer) only partial shielding of the BBSN occurs. However, the longer $x_{T E} / D_{j}=10$ surface, which extended beyond the shock cell train, produced a greater shielding effect.

Similarly, the shock cells imaged for the under-exanded isolated jet and the jet with the $x_{T E} / D_{j}=10$ surface extend to approximately $x / D_{j}=14$ (Figs. $7(\mathrm{c})$ and $9(\mathrm{~b})$, respectively). Figure 11 shows the shock cell structure and the corresponding far-field acoustic data for the under-expanded jet with a $x_{T E} / D_{j}=15$ surface. This longer surface extends to approximately the same axial location as the extent of the shock structures, however, once again the BBSN is only partially shielded at $\theta=60^{\circ}$ and $\theta=90^{\circ}$. The shock cells may extend further downstream than shown, however, the BOS system may not have been sensitive enough to detect the magnitude of the image displacements resulting from these shocks cells. Nevertheless, these shock cells would be relatively weak compared to the ones upstream, but still may be contributing to the BBSN. Therefore, based on the observation that the longer surfaces in both the over-expanded and under-expanded regimes resulted in greater noise shielding, it is concluded that the surface must be several diameters longer than the length of the shock cell train for complete BBSN shielding to occur. 


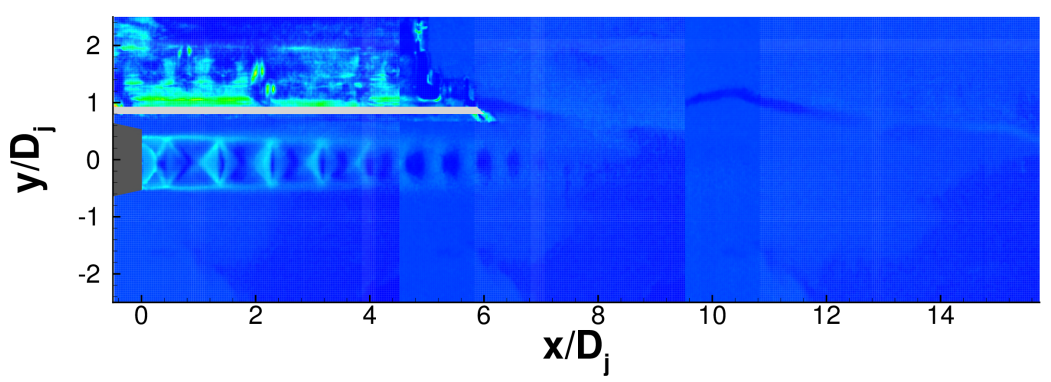

(a) BOS

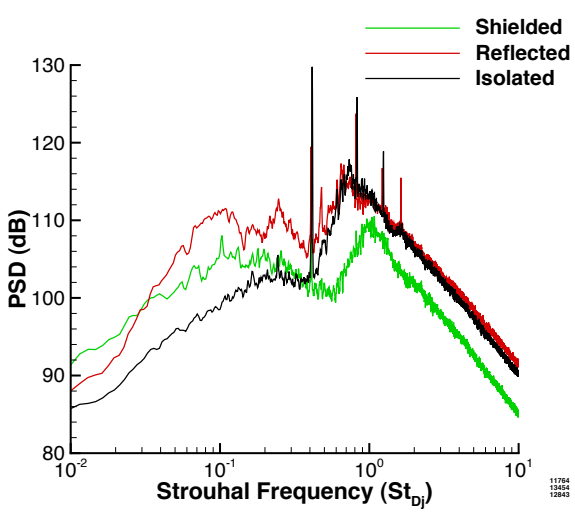

(b) $\theta=60^{\circ}$

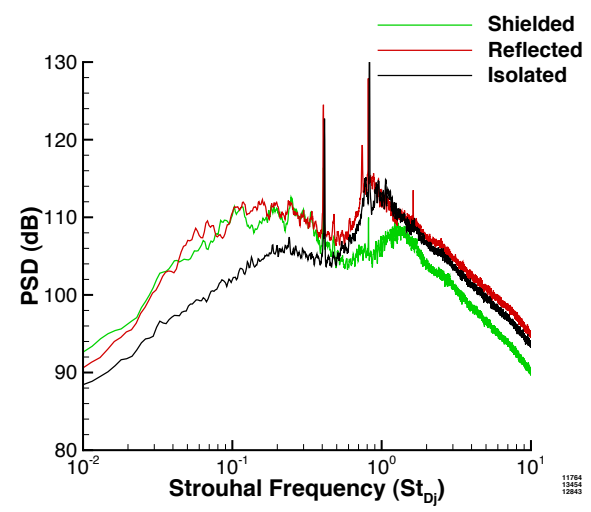

(c) $\theta=90^{\circ}$

Figure 10. BOS and far-field noise measured in an over-expanded jet with a surface at $x_{T E} / D_{j}=6$, $h / D_{j}=0.75$.

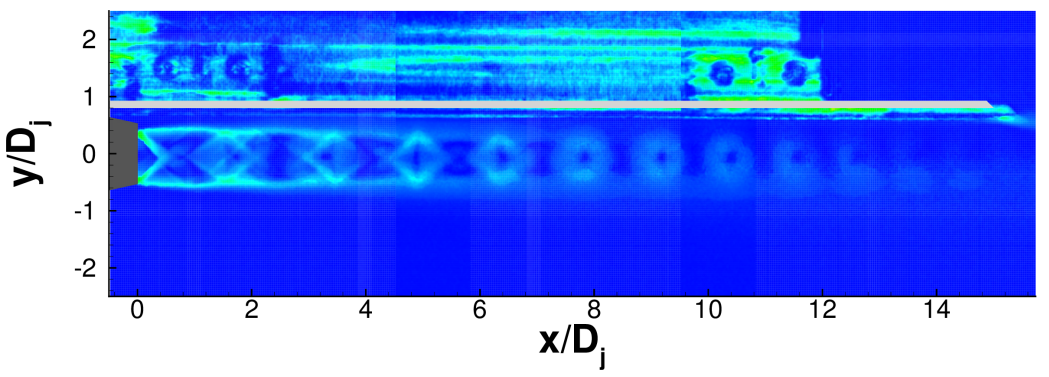

(a) $\mathrm{BOS}$

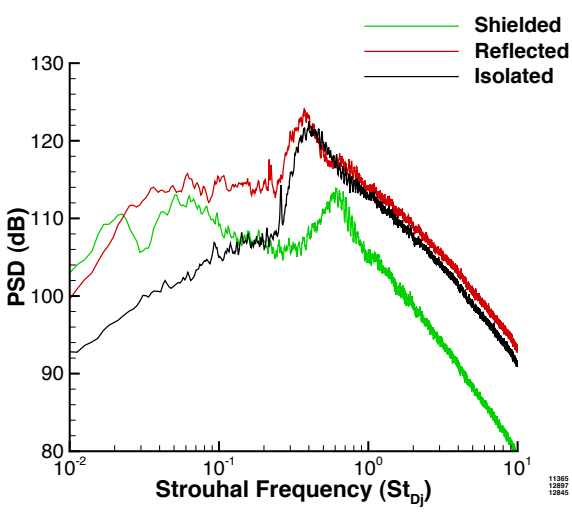

(b) $\theta=60^{\circ}$

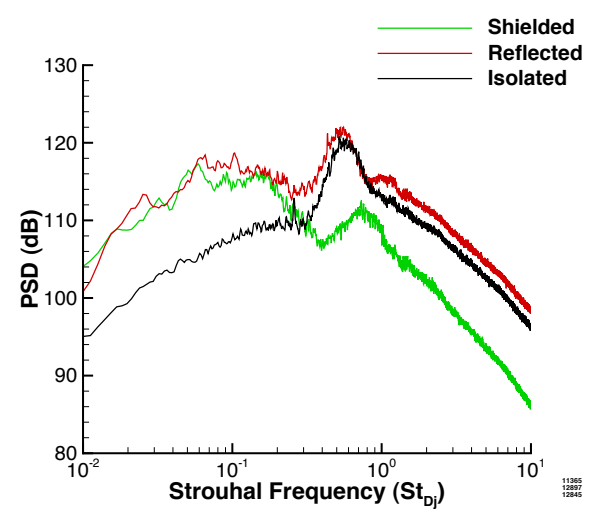

(c) $\theta=90^{\circ}$

Figure 11. BOS and far-field noise measured in an under-expanded jet with a surface at $x_{T E} / D_{j}=15$, $h / D_{j}=0.75$. 


\section{Effect of Radial Surface Distance}

The jet-surface interaction noise and surface shielding effect are strong functions of the radial distance between the jet and the surface. Therefore, the BBSN is also considered as the radial distance to the surface varies. Figure 12 shows the BOS images at the over-expanded jet condition for surfaces at $x_{T E} / D_{j}=6$ and $h / D_{j}=0.5,1.0,1.5,2.0$. (Note, partial BBSN shielding was observed in section 1 for the over-expanded jet with the surface at $x_{T E} / D_{j}=6$ and $h / D_{j}=0.75$.) A careful examination of the BOS images in Fig. 12 gives an indication that the shock cells appear slightly stronger when the surface is at $h / D_{j}=0.5$ (consider the shock cell near $x / D_{j}=4$ for example). Similarly, the shocks may extend a bit farther downstream for $h / D_{j}=0.5$ than for the other radial surface positions (consider the cell near $x / D_{j}=7$ ). However, it is

difficult to identify distinct observational differences in these images. The potential motion of the background as a result of the system translation between reference and data images, may be a contributing factor to any asymmetry present in the BOS images. Therefore, a line profile of the axial image displacement component was extracted along the nozzle centerline from the BOS images, shown in Fig. 13(a), as well as the case of the isolated over-expanded jet, representing the amplitude and the spacing of the shock cell structures at each radial surface distance. These data show that the surface has a minimal impact on the shock cell spacing, even relative to the isolated jet, at points sufficiently upstream of the trailing edge $\left(x / D_{j} \leq 4\right)$. Around the trailing edge, however, the shock cells appear to change spacing and amplitude depending on the radial distance to the surface. This is in contrast to the isolated jet which maintains regular spacing and shows an orderly reduction in amplitude. This disorder around the trailing edge has a small effect on the BBSN as shown in Fig. 13(b). When the surface is present, the BBSN is very similar across all the radial positions, and experiences shielding; the isolated jet had greater levels of BBSN. The lowest BBSN frequencies shift steadily higher as the surface moves away from the jet due to the surface shielding. This indicates that the BBSN reduction is primarily due to noise shielding rather than changes in the shock cells at this condition.

Similarly, Fig. 14(a) shows the axial image displacement component, extracted along the nozzle centerline, depicting the amplitude and the shock cell spacing for the under-exanded jet condition for both the isolated jet and for the surface $x_{T E} / D_{j}=15$ at $h / D_{j}=0.5,1.0,1.5,2.0$. (Note, partial BBSN shielding was also observed in section 1 for the under-expanded jet with the surface at $x_{T E} / D_{j}=15$ and $h / D_{j}=0.75$.) These data clearly show that the surface changes the shock cell structures when the surface is at the closest position $\left(h / D_{j}=0.5\right.$, nozzle lipline). Particularly, the shock cells appear to be spaced further apart and persist farther downstream with a higher amplitude at this radial surface position. The shock cell structures for the other radial surface locations appear to degrade farther downstream and maintain a similar spacing as the isolated jet. Fig. 14(b) shows the impact on the corresponding BBSN, which is significantly higher at $h / D_{j}=0.5$ than at the other radial positions. The BBSN for the $h / D_{j}=0.5$ surface position is also higher than what was previously seen at the $h / D_{j}=0.75$ position shown in Fig. 11(b).

The surface shielding effect at upstream angles typically increases (i.e. lower BBSN) as the surface moves away from the jet and effectively becomes larger to the observer. However, the BBSN in Fig. 14(b) is elevated at $h / D_{j}=2$ relative to $h / D_{j}=1$ and $h / D_{j}=1.5$. This suggests that the shock cells, and thus the BBSN sources, are disrupted by the presence of a surface in this intermediate range, However, there is no evidence in the BOS data that this is occurring to a greater extent at $h / D_{j}=1$ or $h / D_{j}=1.5$ than at $h / D_{j}=2$ so this behavior is noted at this point without a clear cause. 


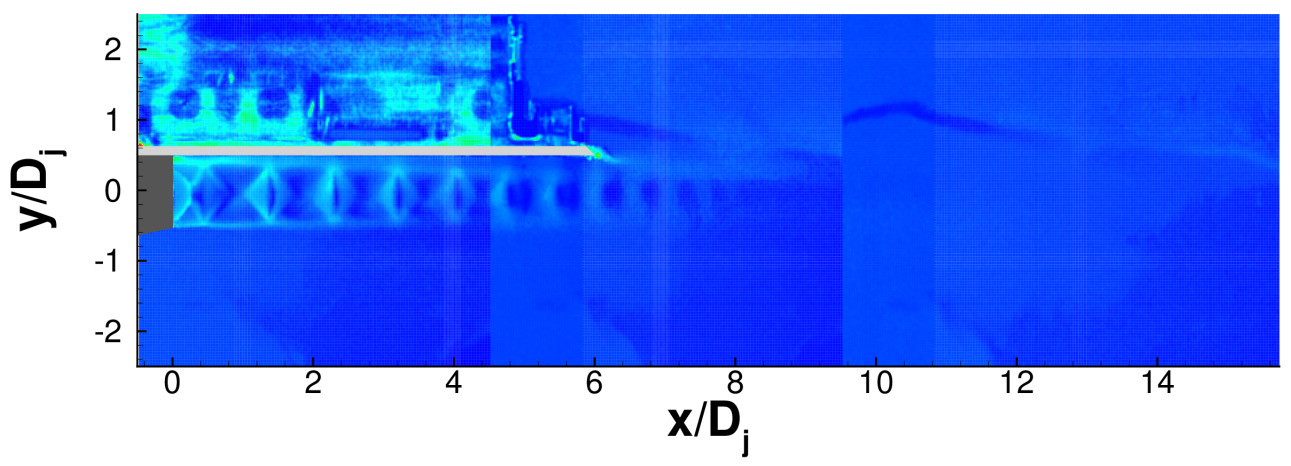

(a) $h / D_{j}=0.5$ (nozzle lipline)

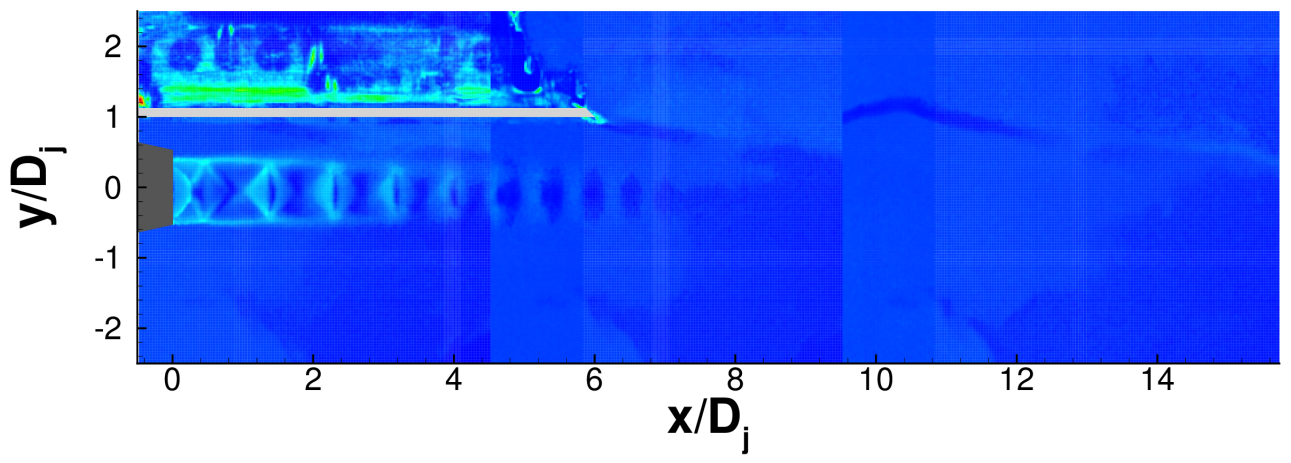

(b) $h / D_{j}=1.0$

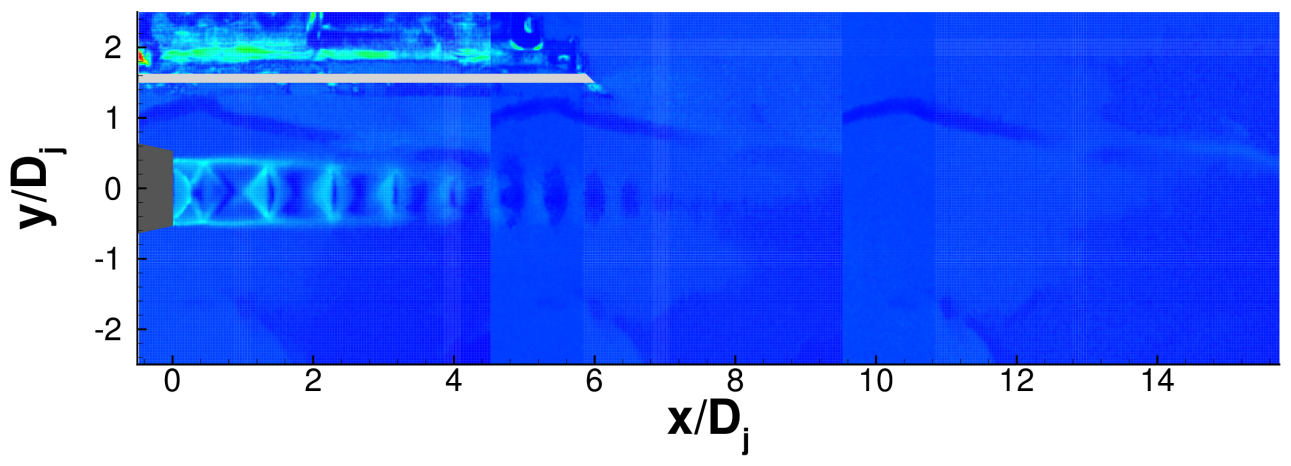

(c) $h / D_{j}=1.5$

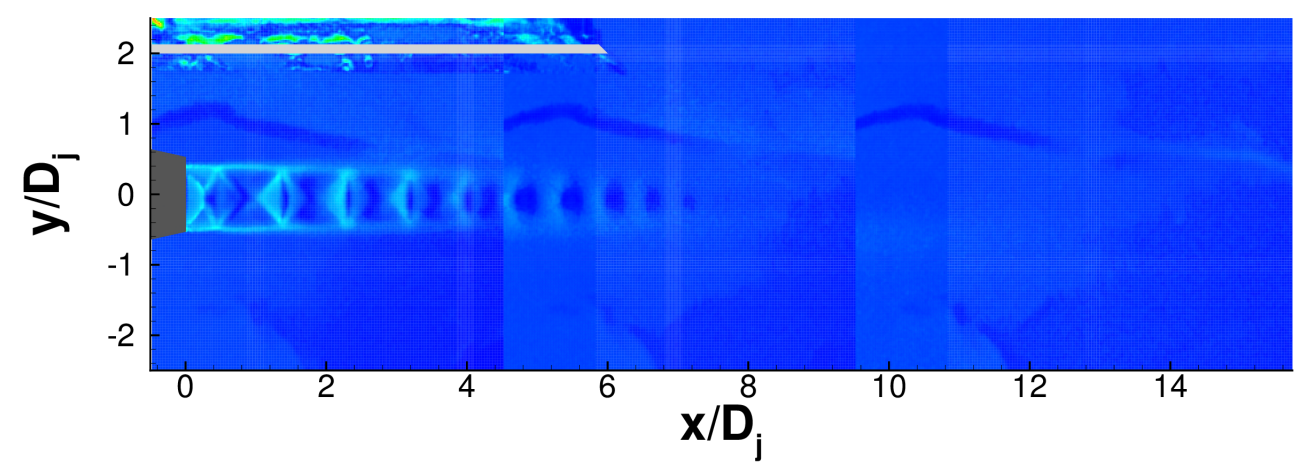

(d) $h / D_{j}=2.0$

Figure 12. BOS images at the over-expanded jet condition with a surface length $x_{T E} / D_{j}=6$ and $h / D_{j}=0.5,1.0,1.5,2.0$. 


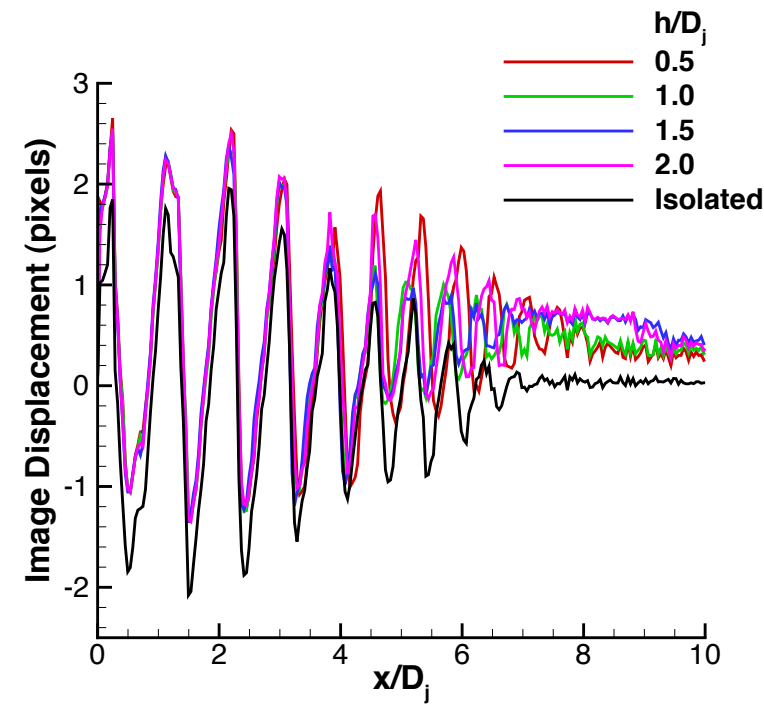

(a) BOS

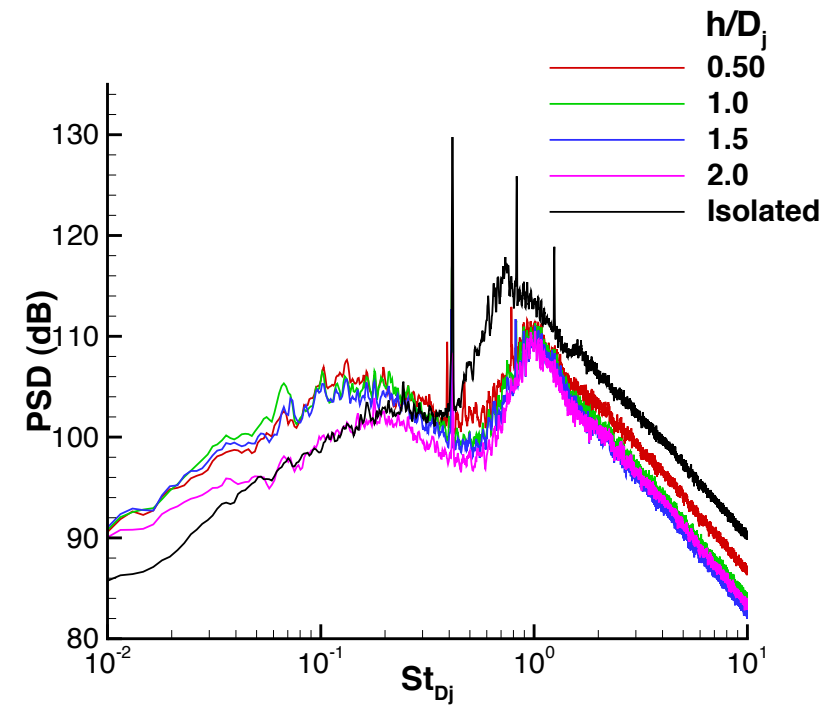

(b) Noise at $\theta=60^{\circ}$

Figure 13. Line profile of the axial image displacement component, representing the amplitude and spacing of the shock cell structures, extracted along the nozzle centerline from the BOS images and far-field noise measured at the over-expanded jet condition with a surface length $x_{T E} / D_{j}=6$ and $h / D_{j}=0.5,1.0,1.5,2.0$.

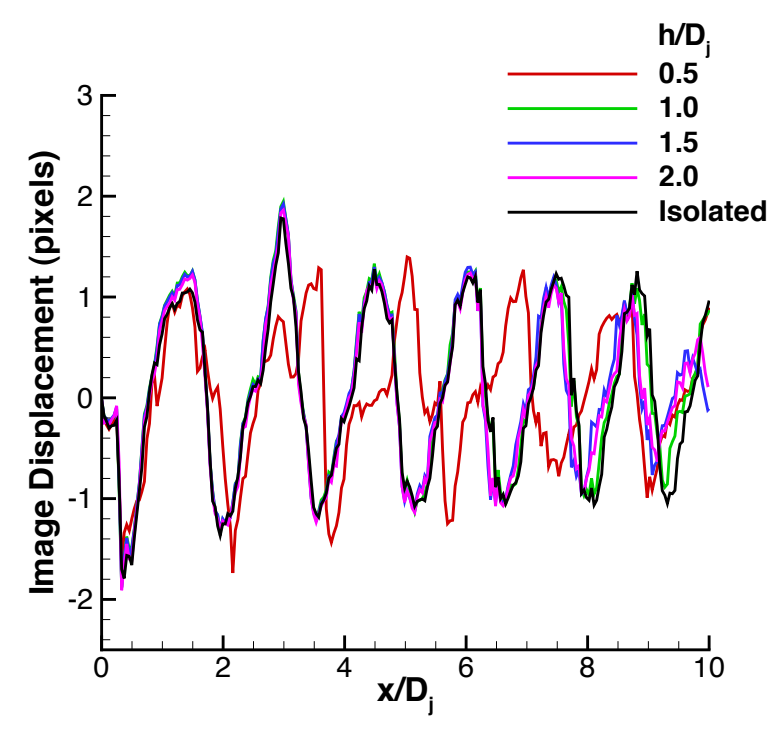

(a) BOS

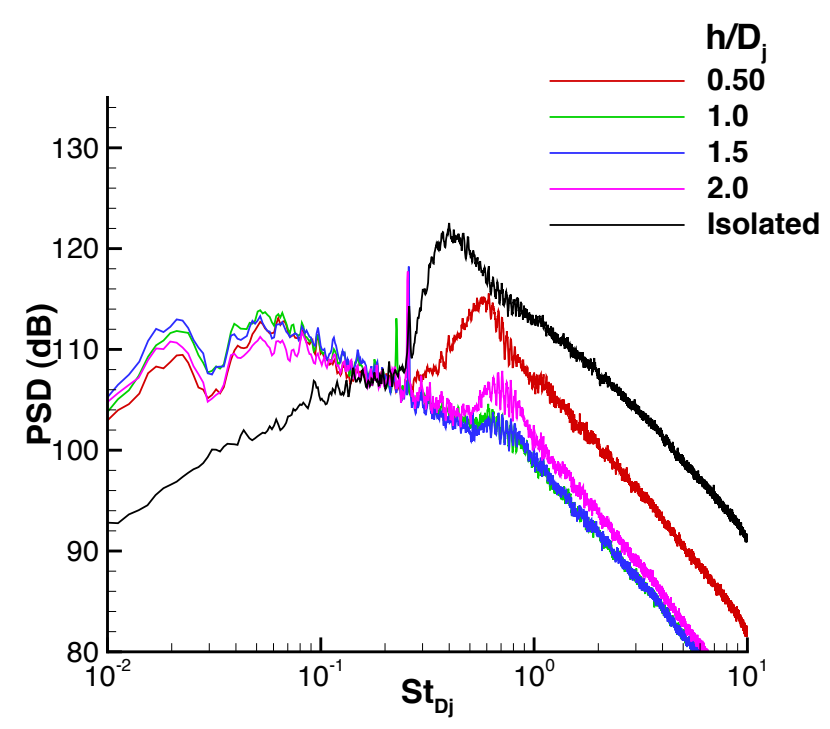

(b) Noise at $\theta=60^{\circ}$

Figure 14. Line profile of the axial image displacement component, representing the amplitude and spacing of the shock cell structures, extracted along the nozzle centerline and and far-field noise measured at the under-expanded jet condition with a surface length $x_{T E} / D_{j}=15$ and $h / D_{j}=0.5,1.0,1.5,2.0$. 


\section{Conclusions}

Background Oriented Schlieren (BOS) was implemented in Phase 2 of the Jet Surface Interaction Test in the Aero-Acoustic Laboratory at the NASA Glenn Research Center. Previous Phase 1 phased array data and far-field acoustic data showed that the Broadband Shock Noise (BBSN) was reduced by the surface when the jet was operated at the over-expanded condition. BOS was implemented in order to investigate whether this reduction in the BBSN was due a change in the shock cell structure caused by an interaction of the planar surface with the high-speed jet flow. BOS data were acquired for under-expanded, ideally-expanded, and over-expanded flow regimes for multiple axial and radial positions at three surface lengths and compared to the previously acquired, corresponding far-field acoustic data. These data were analyzed to study how the shock cell structure and BBSN are affected by a nearby surface. The following observations were made:

(1) The shock cell structure is affected by surfaces close to the jet, particularly when the jet is underexpanded.

(2) Changes to the shock cell structure have a smaller impact on the BBSN compared to the surface shielding effect.

(3) BBSN may be shielded by surfaces close to the jet if those surfaces are sufficiently longer than the shock cell train.

The current generation of noise prediction tools are not equipped for the proposed designs for the next generation of supersonic military and civilian aircraft, which incorporate engines that exhaust near airframe surfaces. These observations from the experimental results of the interaction of the planar surface with the high-speed jet flow, may aid in the development and validation of a supersonic engine exhaust noise prediction tools for the challenge posed by the integrated aircraft designs.

\section{Acknowledgements}

This work was supported by the NASA Fundamental Aeronautics Program, Fixed Wing Project.

\section{References}

\footnotetext{
${ }^{1}$ Brown, C., "Jet-Surface Interaction Test: Far-Field Noise Results", ASME GT2012-69639, 2012.

${ }^{2}$ Podboy, G., "Jet-Surface Interaction Test: Phased Array Noise Source Localization Results", ASME GT2012-69801, 2012.

${ }^{3}$ Venkatakrishnan, L., and Meier, G.E.A, "Density measurements using the Background Oriented Schlieren Technique," Experiments in Fluids, Vol. 37, 2004, pp., 237-247.

${ }^{4}$ Miller, S.A.E., "The Prediction of Broadband Shock-Associated Noise Using Reynolds-Averaged Navier-Stokes Solutions", Ph. D. dissertation, Pennsylvania State University, Dec. 2009.

${ }^{5}$ Brown, C. and Bridges, J., "Small Hot Jet Acoustic Rig Validation", NASA/TM-2006-214324, 2006. 2005.

${ }^{6}$ Bridges, J. and Brown, C., "Validation of the Small Hot Jet Acoustic Rig for Jet Noise Research", AIAA 2005-2846,

${ }^{7}$ Hargather, M. J. and Settles, G. S., "Recent Developments in Schlieren and Shadowgraph", AIAA 2010-4206, 2010.

${ }^{8}$ Richard, H. and Raffle, M., "Principle and applications of the background oriented schlieren (BOS) method", Measurement Science and Technology, vol. 12, pp. 1576-1585, 2001.

${ }^{9} \mathrm{Head}, \mathrm{R}$. W. and Fisher, M. J., "Jet/Surface Interaction Noise: Analysis Of Farfield Low Frequency Augmentation Of Jet Noise Due to the Presence Of A Solid Shield", AIAA 1976-502, 1976.

${ }^{10}$ Viswanathan, K., Alkislar, M. B., and Czech, M. J., "Characteristics of the Shock Noise Component of Jet Noise", AIAA 2008-2835, 2008.

${ }^{11}$ Lawrence, J. L. T., Axarpeyvand, M., and Self, R. H., "Interaction betweeen a Flat Plate and a Circular Subsonic Jet", AIAA 2011-2745, 2011.

${ }^{12}$ Ffowcs, J. E. and Hall, L.H., "Aerodynamic sound generation by turbulen flow in the vicinity of a scattering half plane", J. Fluid Mech., 40(4), pp. 657-670, 1970.

${ }^{13}$ Zaman, K. B. M. Q., "Noise Characteristics of over-expanded Jets from Convergent-Divergent Nozzles", 46 ${ }^{\text {th }}$ AIAA Aerospace Sciences Meeting, AIAA 2008-25, 2008.
} 